\title{
A New EPMA Method For Fast Trace Element Analysis In Simple Matrices
}

\author{
John J. Donovan ${ }^{1}$ and John T. Armstrong ${ }^{2}$ \\ ${ }^{1 .}$ CAMCOR, University of Oregon, Eugene, OR, 97403 \\ ${ }^{2}$ Carnegie Institution for Science, Geophysical Lab, Washington, DC, NW, 20015-1305
}

Traditionally Electron Probe Micro Analysis (EPMA) has relied upon precise characterization of the continuum intensities adjacent to the emission line of interest using Wavelength Dispersive Spectrometry (WDS) for determination of the background under the peak. Recent improvements including new hardware designs with large area Bragg crystals, new software methods implementing exponential and polynomial interpolations to more accurately characterize the curvature of the background, and aggregated spectrometer signals to improve sensitivity, have enabled the EPMA to attain detection limits as low as 2 to 3 PPM in some materials.[1]

However, these so called off-peak methods are subject to spectral interferences on the off-peak intensities which is why it is normal procedure to characterize possible off-peak interferences by performing careful wavelength scans in the region around the emission peak to avoid not only interfering lines but also continuum artifacts.[1] Unfortunately, even high sensitivity and time consuming wavelength scans may not suffice for some samples where the inhomogeneity of major and/or minor elements may introduce unanticipated off-peak interferences on the specified off-peak positions, which may result in significant inaccuracies in the background determination.

Recent work on a new multi-point background method where multiple high precision off-peak measurements (essentially a sparse high sensitivity wavelength scan) combined with a typical quantitative peak intensity measurement, for subsequent "iterative" determination of the optimum background positions, has been developed for complex matrices where such off-peak interferences are variable in complex materials such as monazite.[2]

One may also employ time saving techniques such as only measuring the off-peak intensities every $\mathrm{N}$ points, sometimes referred to as Nth point backgrounds. But all these trace element techniques require careful interpolation from off-peak measurements to obtain the intensity under the peak. If only we could directly measure the intensity under the peak and avoid the off-peak interpolation entirely!

An alternative background correction method known as the mean atomic number (MAN) background correction has been in use for over 20 years now and although originally designed for monochromators that cannot be "detuned" off-peak, the method has been extended and improved by correcting for continuum absorption. [3] This method has demonstrated not only high precision (because the average atomic number of a specimen is essentially a constant for replicate measurements), but also accuracy to 100 to 200 PPM in most silicates and oxides.[4]

Therefore by essentially eliminating the variance of one of the terms in the peak minus background subtraction, because the variances of the peak and background combine in quadrature, $\sigma_{P-B}=\sqrt{{\sigma_{P}}^{2}+{\sigma_{B}}^{2}}$, we improve our sensitivity by approximately $1 / 3$ when $\mathrm{P} \approx \mathrm{B}$, and at the same time reduce our total $\mathrm{x}$-ray integration time by $50 \%$ because we are only measuring the on-peak intensities. Having improved precision and reduced acquisition time, accuracy is further improved by use of the "blank" correction method already described in [1]. Results are shown for Ti and $\mathrm{Al}$ in $\mathrm{SiO}_{2}$ in figures 1 and 2. Further trace work on $\mathrm{TiO}_{2}$ and $\mathrm{ZrSiO}_{4}$ matrices are in progress. 


\section{References}

[1] JJ Donovan, HA Lowers, BG Rusk (2011) Improved Electron Probe Microanalysis of Trace Elements in Quartz, American Mineralogist, 96, 274-282

[2] J Allaz, ML Williams, MJ Jercinovic, and JJ Donovan, (in preparation) Multipoint Background Method: Gaining precision and accuracy in electron microprobe trace element analysis. To be submitted to Chemical Geology

[3] JT Armstrong (1988) Quantitative Analysis of Silicate and Oxide Materials: Comparison of Monte Carlo, ZAF, and Procedures, Microbeam Analysis, 239-246

[4] JJ Donovan and T Tingle (1996), An Improved Mean Atomic Number Background Correction for Quantitative Microanalysis, Jour. of Micros. Microa., 1-7

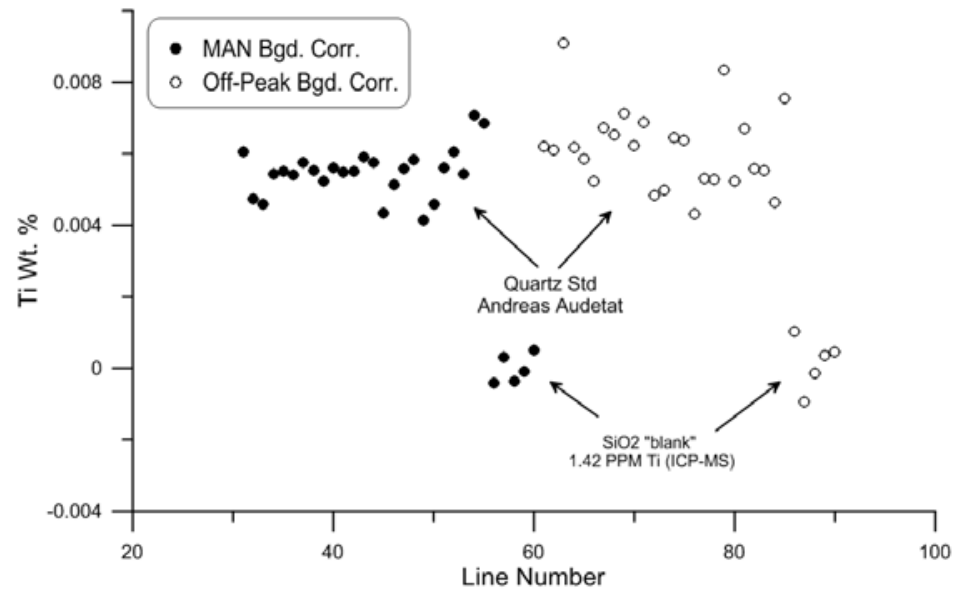

Fig 1. MAN vs. Off-Peak, Ti Ka (LIF/LLIF), $20 \mathrm{keV}, 100$ nA, 10 um, 200 secs on-peak, (200 secs offpeak), Both datasets are aggregates from 2 spectrometers and blank corrected. "Line number" refers to the acquisition order.

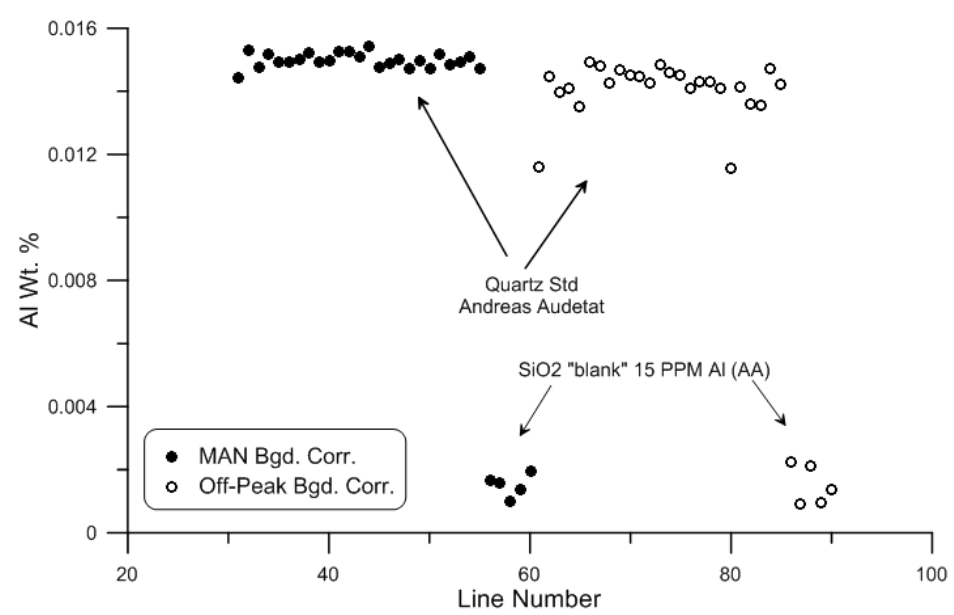

Fig 2. MAN vs. Off-Peak, Al Ka (TAP/LTAP), $20 \mathrm{keV}, 100$ nA, 10 um, 200 secs on-peak, (200 secs off-peak), Both datasets are aggregates from 2 spectrometers and blank corrected. 